\title{
Modeling and Performance Analyses of Hybrid Cellular and Broadcasting Networks
}

\author{
Peter Unger and Thomas Kürner \\ Institute for Communications Technology, Braunschweig Technical University, Schleinitzstrasse 22, 38106 Braunschweig, Germany \\ Correspondence should be addressed to Thomas Kürner, t.kuerner@tu-bs.de
}

Received 1 March 2009; Revised 28 October 2009; Accepted 30 November 2009

Recommended by Sandro Scalise

\begin{abstract}
Mobile communication services are getting more and more important and, in particular, multimedia services have attracted the interest of the users. Mobile TV is one of the most demanded candidates. Powerful and efficient communication systems are needed, which provide high capacities, especially at the downlink. Furthermore, interactivity is essential for supporting the user needs and to extend the service offering. As one possible solution to meet the mentioned requirements, we consider the combination of the cellular network UMTS and the mobile broadcast network DVB-H, which form a hybrid network. We investigate the performance of hybrid networks and develop a system model, which describes the hybrid network and the load switching between both networks. One of the contributions is the definition of the switching bound concept, which represents an efficient tool to assess the necessity and the feasibility of hybrid networks and the amount of load switching. The performance indicators cell load and grade of service are analyzed by using theoretical and realistic scenarios.
\end{abstract}

Copyright ( 2009 P. Unger and T. Kürner. This is an open access article distributed under the Creative Commons Attribution License, which permits unrestricted use, distribution, and reproduction in any medium, provided the original work is properly cited.

\section{Introduction}

With the introduction of the 3rd Generation cellular network Universal Mobile Telecommunications System (UMTS), higher data rates have been made possible and the variety of services has been increased, compared to the previous cellular networks such as General Packet Radio Service (GPRS). In addition to the traditional services, such as voice telephony and messaging, the multimedia services and high data rates have been made possible and accepted by the users. The new offered services, for example, video telephony, Internetbased data communications, and streaming services, make high downlink capacities and efficient networks necessary.

The currently available terminals are equipped with several receivers, which allow for the reception of data from different networks, such as UMTS and DVB-H. Hybrid networks are possible for these types of terminals and the broadcast downlink channel provided by DVB-H can be used very efficiently to serve many users at the same time with high data rate services.

The DVB-H technology is based on the terrestrial digital TV system DVB-T, which has been successfully introduced in many countries $[1,2]$. The signal has been made more robust in order to enable mobile reception with high velocities and at mobile environments, for example, to cope with multipath reception. An additional error protection scheme has been included at the link layer, called Multiprotocol Encapsulation Forward Error Correction (MPE-FEC) [3]. Furthermore, the time slicing concept has been introduced for power saving at the terminal side, while services are transmitted in time slots and the receiver front end can be switched off in the meantime. Furthermore, the time slicing approach enables soft handover and optimized handover algorithms [4], and local content areas in single frequency networks [5]. Depending on the modulation and coding scheme, and the used channel bandwidth, a total downlink data rate of about 6-10 Mbps can be achieved. The individual user data rate depends on the time slicing setup and is typically set to 100 400 kbps.

The currently deployed cellular system UMTS is standardized by the 3rd Generation Partnership Project (3GPP) and makes use of the Wideband Code Division Multiple Access (W-CDMA) technology. All users are served at a common frequency with a $5 \mathrm{MHz}$ bandwidth and are 
separated by orthogonal codes. The network capacity and coverage suffer from interference and have to be planned carefully. The network is deployed in a cellular structure, which is dense in urban environments and larger cells occur in less populated areas. Mobile terminals are assigned to one or more cells, which are established by the Node-Bs. Different bearer services can be used in a circuit or packet switched mode and thus different types of user services are available, such as voice telephony, web browsing, streaming multimedia, and file download.

The combination and the cooperation of both network types has been enabled by the IP Datacast standard, which has been specified within the International DVB Project [6]. It defines the higher layers and form an Internet Protocol(IP-) based end-to-end system, together with the DVB-H specification as the physical layer [7]. In addition to the broadcast downstream, the IP Datacast reference architecture defines an optional link with an interactive cellular network, which is used, for example, for service requests. In terms of hybrid networks, the connection to a cellular network is defined as a mandatory feature.

The combination of both network types has several advantages. On the one hand, popular content, which is requested by several users at the same time, can be switched from UMTS to a broadcast transmission for a more efficient delivery. The UMTS network can be unloaded in order to avoid a cell overload or to reduce the overall cell power and the intercell interference. On the other hand, the broadcast services are enhanced by an uplink channel and new features are possible, such as on-demand services.

Hybrid networks do not exist nowadays. But at this stage, the deployment and operation are possible, since the fully standardized and currently deployed networks UMTS and DVB-H, the IP Datacast standard, and load balancing algorithms as, for instance, proposed in [8] exist. Nevertheless, efficient models are still needed for hybrid network planning and network performance analysis.

We propose a performance model, performance criteria, and constraints for load switching in hybrid network, which is called the switching bound concept. The performance is shown by applying different extents of load switching for a theoretical and a realistic scenario.

This paper is structured as follows. In Section 2 the services and the scenario are defined, which have been considered for our analyses. Section 3 describes models for the individual networks UMTS and DVB-H. In Section 4, the model for hybrid networks is derived, the network performance is evaluated, and the switching bounds are defined. In Section 5, the simulation results are shown by means of a realistic network scenario.

\section{Definition of Services and Scenario}

As mentioned above, several types of services are enabled by UMTS, and eight different types have been summarized in [9]. The defined service set $S$ includes voice and video telephony, web browsing and email, location-based services, short/multimedia massaging service, file download, and streaming multimedia. It is assumed, that some of these

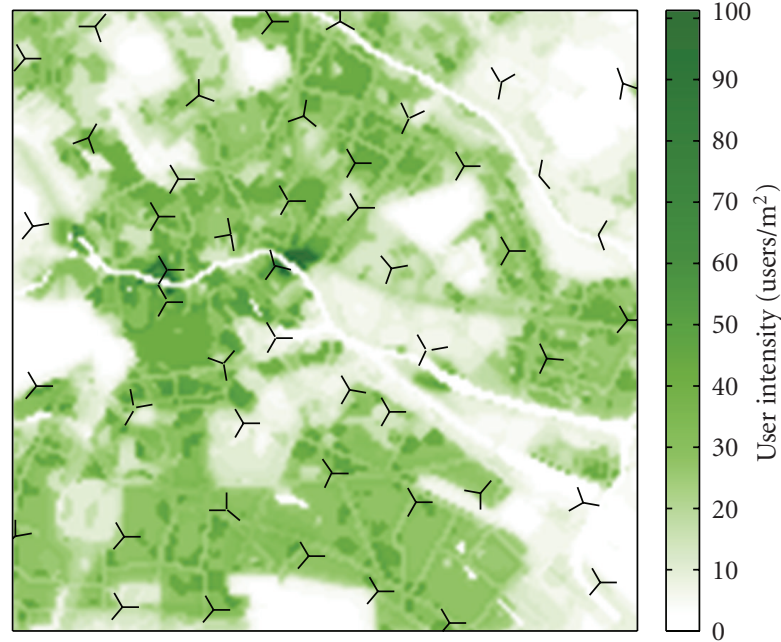

FIGURE 1: UMTS network structure and user intensity for the streaming multimedia service in a $7.5 \mathrm{~km} \times 7.5 \mathrm{~km}$ scenario with a resolution of $50 \mathrm{~m}$. The network is based on the public reference scenario of Berlin available at [10].

services, namely, file download and streaming multimedia, are suitable to be transmitted by both network types, UMTS and DVB-H. For this subset of services, denoted as $S^{(H)}$, load switching can be applied by transferring the transmission from UMTS to the broadcast network. In this paper, we focus on the streaming multimedia service with an average stream data rate of $128 \mathrm{kbps}$. It has been turned out that this service generates a much higher contribution to the cell load, compared to the file download service. The other six services are called cellular services and have always to be delivered by the UMTS network.

In addition to the definition of the service characteristics and the requirements, several scenarios have been proposed in [10], which contain reference network architectures with reference sites, antenna configurations, and predictions of the signal propagation loss. Furthermore, realistic traffic demands have been provided in the form of user intensity maps, which describe the spatial distribution of the service request rate.

We select the public reference scenario of Berlin for our investigation, which is publicly available at [11]. Figure 1 shows the selected UMTS sites and the user intensity of the streaming multimedia service for the entire scenario area. A subset of 48 sites has been selected from the reference scenario, in which the angles of azimuth and elevation have not been modified. However, new signal prediction maps have been calculated with a more sophisticated propagation model, based on [12].

\section{Modeling the UMTS and the DVB-H Network}

The UMTS network is a bidirectional network, which has to be planned for both uplink and downlink. In general, the downlink part is the more critical one, due to a higher amount of traffic demand, caused by asymmetric services. The hybrid network can be beneficial for the downlink part, 
if the additional capacity and the combing capability of the broadcast network are used efficiently. It is assumed that all users are connected to the UMTS network at the uplink, regardless of the way of downlink delivery. Thus, load switching does not effect the uplink and traditional models can be applied. Therefore, we focus on the modeling of the downlink part.

3.1. Modeling the UMTS Downlink. An efficient model for UMTS network evaluation has been proposed in [13] and described in detail in [14]. We review this model in this section, since it is the basis for developing the model for hybrid networks. The UMTS model describes the service requirements, the generated user load, the intracell interference and the interference coupling between cells, which is caused by intercell interference. It provides an efficient estimation of the necessary transmit power for the mobile terminals and the Node-Bs by solving a linear equation system, which is called interference coupling system.

The model can be used in conjunction with snapshot analyses using Monte-Carlo simulations. Several details can be implemented with this approach and thus, it generates accurate results for a high number of snapshots. But it is computational expensive. A more efficient approach has been proposed in [15] and is described more in detail in [14], which uses expected values for the user demand, instead of individual users. Thus, it replaces the computational expensive snapshot approach with an average view on the current network load. The drawback is that the accuracy of the results is somewhat decreased and some details are not modeled, for example, different velocities.

In the context of this work, both methods have been considered. First, the downlink of hybrid networks is modeled and the principal behavior of load switching is shown by means of the expected approach. For network simulations performed in Section 5, a realistic network and the snapshot approach are applied.

For determining the cell load and the coupling between the cells, mobile terminals are assigned to the Node-Bs by the best server approach, whereas that cell is selected, which provides the strongest pilot (CPICH) signal. The best server area $A_{i}$ of cell $i$ is determined by using the pilot channel power $p_{i}^{(\mathrm{CPICH})}$ and the end-to-end channel gain $\gamma_{i}(x)$ from antenna $i$ to location $x$, which basically includes the propagation loss and antenna gains.

Each service requires a specific carrier-to-interferenceand-noise ratio (CINR) target, denoted as $\mu_{s}$, which includes the processing gain of the selected bearer service and the required $E_{b} / N_{0}$ target [13]. The activity of the bearer usage is denoted by $\alpha_{s}$ and the orthogonality loss factor of a location $x \in A_{i}$ is denoted by $\omega(x)$. The orthogonality of the signals is partly lost due to multipath propagation effects and thus, intracell interference is caused. The caused load of service $s$ at location $x$ is calculated by

$$
l_{s}(x):=\frac{\alpha_{s} \mu_{s}}{1+\omega(x) \alpha_{s} \mu_{s}} .
$$

The interference coupling approach defines the coupling factors for cell $i(2)$ as expected values, which form the interference-coupling downlink matrix $\mathbf{C}=\left(c_{i j}\right)_{i, j \in\left\{1, \ldots, N_{\text {cell }}\right\}}$. The parameter $T_{s}(x)$ represents the user intensity of service $s$ at location $x$ :

$$
\begin{aligned}
& c_{i i}=\int_{x \in A_{i}} \omega(x) \sum_{s \in S} l_{s}(x) T_{s}(x) d x, \\
& c_{i j}=\int_{x \in A_{i}} \frac{\gamma_{j}(x)}{\gamma_{i}(x)} \sum_{s \in S} l_{s}(x) T_{s}(x) d x .
\end{aligned}
$$

The coupling system for all cells $i$ can be written as

$$
\bar{p}_{i}=c_{i i} \bar{p}_{i}+\sum_{j \neq i} c_{i j} \bar{p}_{j}+\bar{p}_{i}^{(\eta)}+p_{i}^{(C C)}
$$

with $\bar{p}_{i}^{(\eta)}=\int_{x \in A_{i}}\left(\eta(x) / \gamma_{i}(x)\right) \sum_{s \in S} l_{s}(x) T_{s}(x) d x$ being the average required power to overcome the noise in a non interfered cell. The constant power values of all common channels including the pilot channel are summed up in $p_{i}^{(C C)}$. The parameter $\bar{p}_{i}$ denotes the average transmit power of cell $i$ in order to serve all users and to overcome noise and interference.

The resulting power values $\bar{p}_{i}$ must not exceed the maximum feasible power $p_{\max }$. In order to avoid cell overloading caused by high traffic demand and high interference, the scaling factor $\lambda_{i} \in[0,1]$ has been proposed in [14]. It is used to scale the coupling matrix with $\operatorname{diag}(\lambda) \mathbf{C}$ in order to reduce the traffic demand per cell and the coupling to the other cells. This approach assumes perfect load control with applying an exact downgrade of cell load. The scaling factor $\lambda_{i}$ represents the Grade of Service (GoS); that is, the fraction of served traffic compared to the offered traffic in cell $i$, and $\left(1-\lambda_{i}\right)$ defines the blocking probability of cell $i$. In [14] an iterative process has been defined to estimate the parameters $\lambda_{i}$ and $\bar{p}_{i}$ for each cell. The interference coupling concept assumes perfect power control, which enables to precisely assign the necessary power to each user.

Another measure of the quality in network planning is the average other-to-own interference $\bar{l}_{i}$, which describes the total intercell interference to cell $i$ (4). Note that this definition has to be divided by the average orthogonality loss (e.g., $\bar{\omega}=0.673$ for urban environments) in order to be comparable to traditional definitions, for example, in [13]:

$$
\bar{\iota}_{i}=\frac{\sum_{j \neq i} c_{i j} \bar{p}_{j}}{c_{i i} \bar{p}_{i}} .
$$

In [14], the so-called pole equations have been defined to calculate the necessary average transmit power $\bar{p}_{i}(5)$ and the grade of service $\lambda_{i}^{(C)}(6)$ per cell with a given other-toown interference. We have used the notation ${ }^{(C)}$ to denote 
the cellular network, since we have also defined the GoS criteria for the broadcast ${ }^{(B)}$ and the hybrid ${ }^{(H)}$ network:

$$
\begin{aligned}
& \bar{p}_{i} \\
& = \begin{cases}\frac{p^{(C C)}+\bar{p}_{i}^{(\eta)}}{1-\left(1+\bar{l}_{i}\right) c_{i i}} & \text { if }\left(1+\bar{l}_{i}\right) c_{i i}<\frac{p_{\max }-p^{(C C)}-\bar{p}_{i}^{(\eta)}}{p_{\max }}, \\
p_{\max }, & \text { otherwise, }\end{cases}
\end{aligned}
$$

$$
\begin{aligned}
& \lambda_{i}^{(C)} \\
& = \begin{cases}1 & \text { if }\left(1+\bar{l}_{i}\right) c_{i i}<\frac{p_{\max }-p^{(C C)}-\bar{p}_{i}^{(\eta)}}{p_{\max }}, \\
\frac{p_{\max }-p^{(C C)}}{p_{\max }\left(1+\bar{l}_{i}\right) c_{i i}+\bar{p}_{i}^{(\eta)}}, & \text { otherwise. }\end{cases}
\end{aligned}
$$

The transmit power $\bar{p}_{i}$ and the GoS $\lambda_{i}^{C}$ form a complementary system. The transmit power increases until the offered traffic load can be served or the maximum power is reached. Additional traffic load is blocked, which reduces the GoS of the cell. In this work, alternative concepts of solving a network overload as described in [16] are not considered.

The GoS of the entire UMTS network $\bar{\lambda}^{(C)}$ is estimated by a weighting sum, considering the offered traffic load $\tau_{i}=$ $\int_{x \in A_{i}} \sum_{s \in S} l_{s}(x) T_{s}(x) d x:$

$$
\bar{\lambda}^{(C)}=\frac{\sum_{i} \tau_{i} \lambda_{i}^{(C)}}{\sum_{i} \tau_{i}} .
$$

The utilization of the transmit power in each cell is measured with the downlink cell load. It is defined by the ratio of the necessary downlink transmit power compared to the total available maximum power: $L_{i}^{(C)}=\bar{p}_{i} / p_{\max }$.

3.2. Modeling the Broadcast Network. In contrast to the UMTS network, the required system capacity of broadcast network is independent on the actual number of users. The necessary amount of capacity depends on the number of different content items, that are requested by the users [17].

The requests for different content have different probabilities; that is, some items are more popular compared to others. A common description of the popularity is the Zipf-distribution defined in (8). Detailed analyses and measurements have been performed and described in [18].

The probability that a user request corresponds to item $i$ is defined as

$$
P(X=i)=\frac{1}{\Omega} i^{-\kappa} \quad \text { with } \Omega=\sum_{i=1}^{N_{\text {item }}} i^{-\kappa} .
$$

The parameter $N_{\text {item }}$ represents the number of items offered by the content provider and $\kappa \geq 0$ is the shape parameter of the popularity distribution. For a value of $\kappa=0$, the requests are uniformly distributed to all items. For an increasing value of $\kappa$, the popularity concentrates more and more on specific items.

As mentioned above, we consider the streaming multimedia service for hybrid delivery, whereas several requested streams are switched to the broadcast network. Since streaming is a real-time service, the broadcast capacity is shared among all switched streams in a parallel transmission. Each stream requires a constant data rate $d_{i}$ in order to guarantee sufficient Quality of Service (QoS).

We define the broadcast cell load $L^{(B)}$ in (9), which depends on the number of delivered streams and the capacity $D^{(B)}$, reserved for switchable services. If $L^{(B)}$ exceed one, the broadcast cell is overloaded, some streams have to be blocked and the corresponding users cannot be served:

$$
L^{(B)}=\sum_{i=1}^{n} \frac{d_{i}}{D^{(B)}} .
$$

The maximum number of streams, which can be delivered in parallel, is calculated by $n_{\max }=\left\lfloor D^{(B)} / \bar{d}\right\rfloor$ with $\bar{d}$ being the average required data rate of all streaming services.

\section{Modeling Hybrid Networks}

In a hybrid system, the delivery of services can be switched between the downlinks of both networks, UMTS and DVB$\mathrm{H}$. The overall network performance can be optimized with properly applying load switching.

The load switching in hybrid networks is modeled by specifying the amount of traffic, which is switched from the UMTS network to the broadcast network. In contrast to GoS scaling, the switched traffic is not lost, but served by the broadcast system. Thus, the UMTS network is unloaded, while improving the overall grade of service.

First of all, the offered traffic has to be classified into the cellular service demand and switchable services (see Section 2). Since the coupling matrix $\mathbf{C}$ is generated by summing up the user demand for each service, this matrix can be split into the cellular $\mathbf{C}^{(C)}$ and the hybrid part $\mathbf{C}^{(H)}$. All elements in the matrices can be calculated by using (2) and the appropriate set of services $S / S^{(H)}$ and $S^{(H)}$.

We define the load share parameter $\beta$, which specifies the fraction of cellular (nonswitchable) traffic load as $\beta=$ $c^{(C)} /\left(c^{(C)}+c^{(H)}\right)$. For $\beta=0$, it is possible to switch the entire traffic load to the broadcast network and for $\beta=1$, no switchable traffic load exists at all. The ratio parameter $\beta$ is dependent on the location. An average value of about $43 \%$ occurs for evaluating the area of the Berlin reference scenario by considering the streaming multimedia service for load switching. That means, about $57 \%$ of the traffic can be used for unloading the UMTS network.

4.1. Definition of Load Switching. The fraction of traffic load is estimated, which is actually needed to be switched to the broadcast network for a successful unloading of the UMTS network. For example, a typical target criteria for network planning is to achieve a cell blocking rate of $2 \%$ or less, which corresponds to $\lambda_{i} \geq 0.98$. 


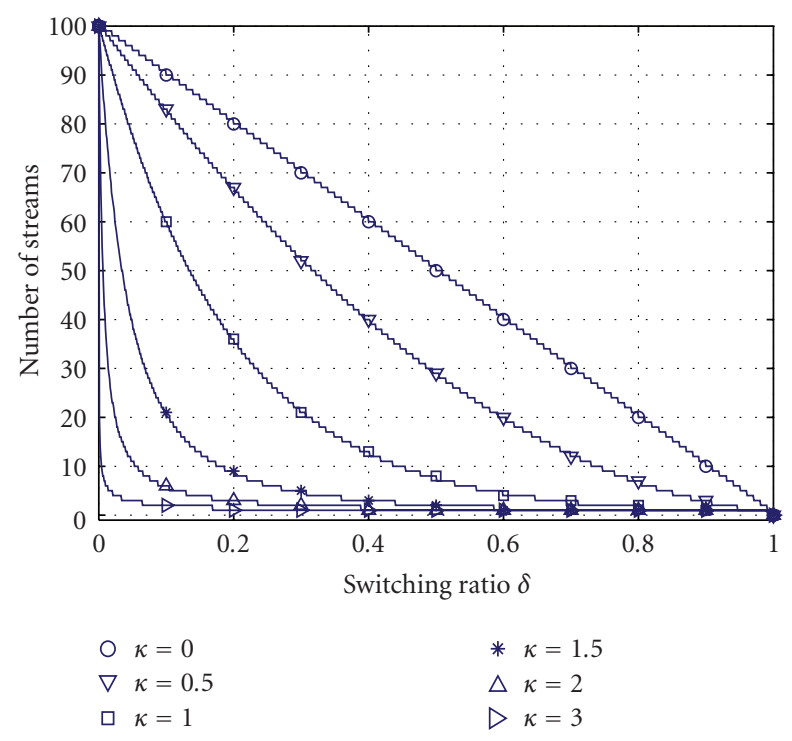

Figure 2: Comparing the switching parameter $\delta$ with the number of streams $n$, which are offered to the broadcast system, depending on the popularity distribution.

We define the switching parameter $\delta_{i} \in[0,1]$, which is used to control the amount of traffic load, switched between both networks. A value of $\delta_{i}=0$ indicates that all requests for the switchable service are switched to the broadcast network. For example, all streaming services are served by the DVB-H network. Therewith, we define $\tilde{c}_{i j}$ as the new coupling factor for the UMTS network with

$$
\tilde{c}_{i j}=c_{i j}^{(C)}+\delta_{i} c_{i j}^{(H)} .
$$

It has been shown in [8] that those service items are beneficial for optimizing hybrid networks, which causes a high load in the UMTS network. The product of arrival rate and required resources (i.e., data rate) has been taken as a measure to define the switching order. In this work, we assume equal data rates for all streams and therefore, the item request rate is directly proportional to its popularity. Thus, we assume that the most popular items are switched first.

As mentioned before, both networks can be described by different schemes, the user and content dependence. In order to estimate the broadcast cell load, we have to transform the applied load switching with the switching parameter $\delta$ into the corresponding number of streams $n_{\delta}$, which have to be delivered by the broadcast network. The fraction $(1-\delta)$ corresponds to the $n$ most popular items. Thus, we look for the minimum $n$, which fulfils $1-\delta \leq P(X \leq n)$. In other words, the $\left(N_{\text {item }}-n\right)$ low popular items have to be served by the UMTS network. Thus, the relation between $n$ and $\delta$ can be defined as

$$
n_{\delta}=\min _{n}\{n \mid P(X>n) \leq \delta\} .
$$

On the other hand, the estimation of $\delta$ for a given number of streams $n$, which have to be switched, can be described by $\delta_{n}=P(X>n)$.
In Figure 2, the number of streams is shown, which is offered to the broadcast system, depending on $\delta$ and the popularity shape parameter $\kappa$. The total number of items is set to $N_{\text {item }}=100$. It can be seen that, with $\kappa=0$, all items have the same popularity and thus, $n$ increases linearly for more broadcast traffic. For an increasing $\kappa$, a higher fraction of traffic corresponds to the more popular items. The broadcast technology allows for combining user requests to the corresponding high popular items. Thus, for the same $\delta$, the broadcast network is less loaded for higher values of $\kappa$.

4.2. Definition of Switching Bounds. Depending on the system parameters and the traffic demand, the possible range of switching may be limited, if cell load values of $L^{(B)} \leq 1$ and $L^{(C)} \leq 1$ have to be guaranteed. We introduce the switching bound concept, which defines a bound for $\delta$ for each network separately. In order to apply reasonable load switching in a hybrid network, the broadcast network should define the lower and the UMTS network the upper bound of $\delta$. The switching bounds indicate the necessity and feasibility of applying a hybrid network. It is obvious, if no bound has been set by the UMTS network, no unloading is required.

4.2.1. Broadcast Bound. The bound $\delta^{(B)} \in[0,1]$ is defined by the parameters of the broadcast network and the streaming service. Since the broadcast capacity is limited, the number of parallel transmittable streams is limited too. If the broadcast cell load defined in (9) equals one, the maximum number of streams $n_{\max }$ is reached. The broadcast bound $\delta^{(B)}$ is calculated by

$$
\delta^{(B)}=P\left(X>n_{\max }\right)=P\left(X>\left\lfloor\frac{D^{(B)}}{\bar{d}}\right\rfloor\right) .
$$

Therefore, $\delta^{(B)}$ depends on the broadcast capacity $D^{(B)}$, the service data rate $d_{i}$, and the popularity distribution, that is, $N_{\text {item }}$ and $\kappa$.

Figure 3 shows $\delta^{(B)}$ depending on the popularity shape parameter $\kappa$ and the broadcast cell capacity $D^{(B)}$. The number of items is set to $N_{\text {item }}=100$ and the average streaming data rate is $\bar{d}=128 \mathrm{kbps}$. It is obvious that for higher broadcast data rates, more traffic can be switched to the broadcast network and $\delta^{(B)}$ is decreased and less restrictive. For a more even popularity distribution (smaller values of $\kappa$ ), the bound increases due to a higher variety of the requested streams and thus, a higher broadcast capacity demand. For a very large values of $\kappa$, the popularity corresponds to a very small number of items, in extremum to one single item. Thus, all requests can be delivered by the broadcast network. For $D^{(B)}=N \bar{d}=12.8 \mathrm{Mbps}$, it is possible to serve all items by the broadcast network, thus $\delta^{(B)}=0$ and no restrictions exist from the broadcast network side. For a too small capacity, that no stream can be transmitted, the broadcast bound equals one for any value of $\kappa$.

4.2.2. Cellular Bound. The cellular bound $\delta_{i}^{(C)} \in[0,1]$ describes the minimum necessary unloading of cell $i$ by switching streaming services to the broadcast network, until 


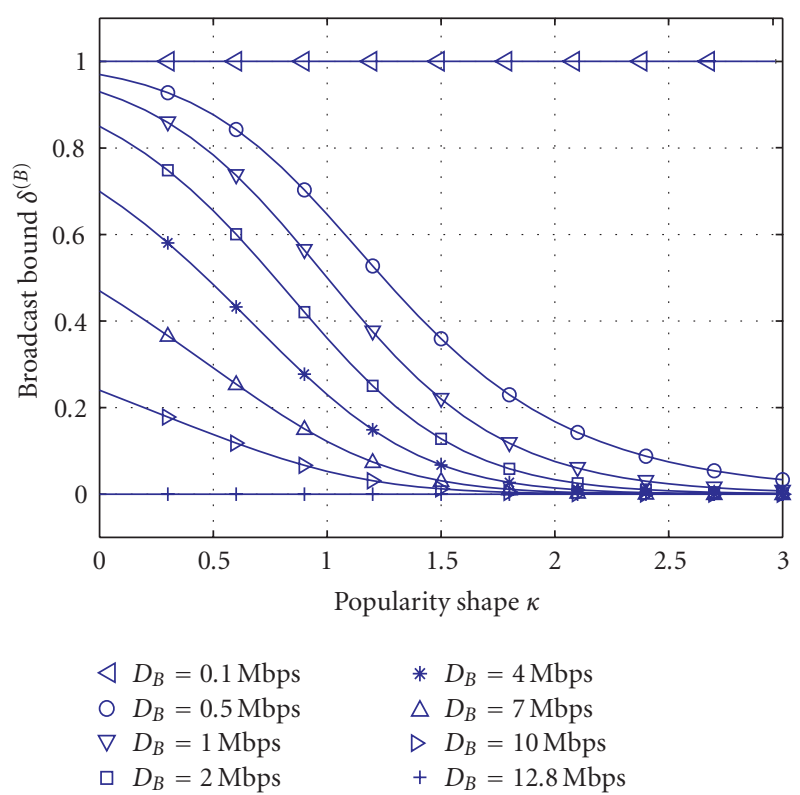

FIGURE 3: Broadcast bound $\delta^{(B)}$ depending on the popularity shape $\kappa$ and the total broadcast capacity.

the GoS target $\hat{\lambda}$ is reached. In this work, we use $\hat{\lambda}=0.98$. Once the cell is able to handle the amount of offered traffic, a further unloading yields to a reduction of the required transmit power $\tilde{p}_{i}$. In order to estimate $\delta^{(C)}$, we use (6) with $\tilde{c}_{i i}=c_{i i}^{(C)}+\delta_{i} c_{i i}^{(H)}$ and $\tilde{p}_{i}^{(\eta)}=p_{i}^{(\eta, C)}+\delta_{i} p_{i}^{(\eta, H)}$, and transform it to $\delta_{i}$ :

$$
\begin{aligned}
& \delta_{i}^{(C)} \\
& = \begin{cases}\frac{p_{\max }-p^{(C C)}-\hat{\lambda} p_{\max }\left(1+\bar{l}_{i}\right) c_{i i}^{(C)}-p_{i}^{(\eta, C)}}{\hat{\lambda} p_{\max }\left(1+\bar{l}_{i}\right) c_{i i}^{(H)}+p_{i}^{(\eta, H)}}, & \lambda_{i}^{(C)}<\hat{\lambda}, \\
1, & \hat{\lambda} \leq \lambda_{i}^{(C)} \leq 1 .\end{cases}
\end{aligned}
$$

It is obvious that a solution is only possible, if switchable traffic exists in cell $i$, so that $c_{i i}^{(H)}>0$. If all services are switchable $(\beta=0)$ and the GoS target is set to one, the bound $\delta_{i}^{(C)}$ becomes equal to the $\operatorname{GoS} \lambda_{i}^{(C)}$.

In the following, we investigate a theoretical scenario of a single cell, in which the influence from the surrounding cells, that is, the average other-to-own interference $\bar{l}_{i}$, is assumed to be constant. The curves in Figure 4 depict the cellular bound $\delta^{(C)}$. The bound is shown for different cell loads $c_{i i}$ and depending on load share parameter $\beta_{i i}$. For $\beta_{i i}=1$, no hybrid traffic exists and no load switching can be applied. For $\beta_{i i}=0$, all services can be transmitted by both network types. This case shows the less restrictive bound values, since no cellular background traffic exists.

In this example, the following parameters have been assumed: $p_{\max }=14 \mathrm{~W}, p^{(C C)}=4 \mathrm{~W}, \bar{l}_{i}=0.6$, and $p^{(\eta)}=$ $0.96 \mathrm{~mW}$. If $\lambda_{i}^{(C)} \geq \hat{\lambda}$, no traffic needs to be switched, since the QoS target is already achieved without applying

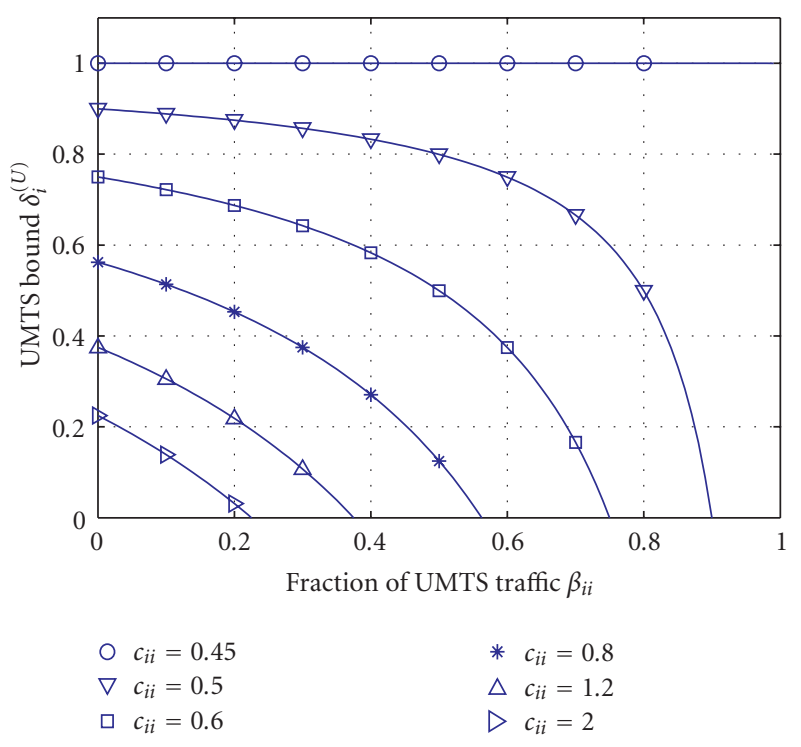

FIGURE 4: Cellular bound $\delta_{i}^{(C)}$ for cell $i$ depending on the load share parameter $\beta$ and the total offered traffic load.

unloading. The threshold in our case is $c_{i i}=0.45$. This threshold can be calculated by using (6) with $\lambda_{i}^{(C)}=\hat{\lambda}$. Thus, $\delta_{i}^{(C)}$ is set to one for all values of $c_{i i}$, which are lower or equal as this threshold.

In general, it can be seen that $\delta_{i}^{(C)}$ decreases for higher cell load, since the cell needs to be unloaded more and more. For high traffic loads, the proportion of switchable traffic needs to be high, that is, low values of $\beta$, in order to apply sufficient load switching for successfully unload the cells.

4.2.3. Discussion on Switching Bounds. We have defined two bounds for the load switching, which depend on the offered traffic, the load share, and on the parameters of the broadcast and UMTS network, respectively. The broadcast bound is valid for the entire broadcast cell. Each UMTS cell defines an individual bound, depending on the current loading, the grade of service, and the cell parameters.

In order to successfully apply unloading in a hybrid network the broadcast bound needs to be lower than the cellular bound, $\delta^{(B)} \leq \delta^{(C)}$. All UMTS cells, which are covered by a broadcast cell and which are considered for a hybrid mode, need to be evaluated. Therefore the minimum of all $\delta_{i}^{(C)}$ has to be considered, if the quality target has to be reached. Nevertheless, strategies for network planning and estimating the appropriate cellular bound are beyond the scope of the paper.

If no broadcast bound is defined, the necessary broadcast data rate can be determined. The estimated switching parameter $\delta$ is based on the evaluation of the UMTS cells. It defines the necessary capacity of the broadcast network, which can be estimated by the number of streams to be transmitted (11). By summing up the required data rates of all items switched to broadcast the necessary broadcast data rate can be calculated. 
4.3. Grade of Service in Hybrid Networks. The grade of service of a broadcast network can be defined in two ways, a contentor a user-based description. The user-based definition has been selected, due to compatibility reasons to the cellular network and for a common GoS estimation of hybrid networks. It is estimated by evaluating the probability of the blocked streams.

If $\delta<\delta^{(B)}$, the capacity of the broadcast network is not sufficient for transmitting the switched content and users have to be blocked. The probability of not served streams is

$$
\begin{aligned}
P\left(X \leq n_{\delta}\right)-P\left(X \leq n_{\max }\right) & =(1-\delta)-\left(1-\delta^{(B)}\right) \\
& =\delta^{(B)}-\delta .
\end{aligned}
$$

Thus the grade of service of the broadcast network is calculated by $\lambda^{(B)}=\min \left\{1,1-\left(\delta^{(B)}-\delta\right)\right\}$. It is obvious that for $\delta \geq \delta^{(B)}$, no blocking occurs and $\lambda^{(B)}=1$.

In order to estimate the grade of service $\lambda_{i}^{(H)}$ for the hybrid network, the GoS parameters $\lambda_{i}^{(C)}$ and $\lambda^{(B)}$ have to be combined. Equation (15) describes the ratio of the traffic served by the hybrid network compared to the total offered traffic. This ratio is transformed into a weighted term; whereas the weights describe the fraction of cellular and switchable traffic:

$$
\begin{aligned}
\lambda_{i, \delta}^{(H)} & =\frac{\tilde{\lambda}_{i, \delta}^{(C)}\left(c_{i i}^{(C)}+\delta c_{i i}^{(H)}\right)+\lambda_{\delta}^{(B)}(1-\delta) c_{i i}^{(H)}}{c_{i i}} \\
& =\left[\delta\left(1-\beta_{i i}\right)+\beta_{i i}\right] \tilde{\lambda}_{i, \delta}^{(C)}+(1-\delta)\left(1-\beta_{i i}\right) \lambda_{\delta}^{(B)} .
\end{aligned}
$$

For $\delta=1$, the total traffic has to be transmitted by the UMTS network, thus, $\lambda^{(H)}=\lambda^{(C)}$. In the case that $\delta=0$, the UMTS network is unloaded by all switchable traffic, but this traffic is partly lost due to an overloaded broadcast network. The GoS of the broadcast network results in $\lambda^{(B)}=1-\delta^{(B)}$. The GoS of the UMTS network can be calculated by (6) using $\widetilde{c}_{i i}=c_{i i}^{(C)}$ and $\tilde{p}_{i}^{(\eta)}=p_{i}^{(U, \eta)}$.

4.4. Performance Analysis. A single cell is considered with fixed interference from the surrounding cells. This simplified scenario is used in order to show the basic behavior of the network performance indicators cell load $\left(L^{(C)}\right.$ and $\left.L^{(B)}\right)$ and grade of service $\left(\lambda^{(C)}, \lambda^{(B)}\right.$, and $\left.\lambda^{(H)}\right)$.

Figure 5 shows these performance indicators. In this example, the parameters of Section 4.2 and the following parameters have been used: $D^{(B)}=2 \mathrm{Mbps}, d_{i}=128 \mathrm{kbps}$, $N_{\text {item }}=100, \kappa=1, c_{i i}=0.5$, and $\beta=0.6$.

The used parameter values result in the bound values of $\delta^{(C)}=0.78$ and $\delta^{(B)}=0.36$. The cell load of the broadcast cell increases with a decreasing $\delta$ until the broadcast bound is reached. The nonuniform steps are caused by the different content popularity values. The UMTS cell load decreases while unloading beyond the cellular bound, caused by a lower necessary transmit power $\tilde{p}_{i}$.

The grade of service is shown for both network types and the hybrid combination. For high values of $\delta$ the curves of $\lambda^{(C)}$ and $\lambda^{(H)}$ are similar due to a small contribution of the broadcast part with a small amount of traffic switched to the

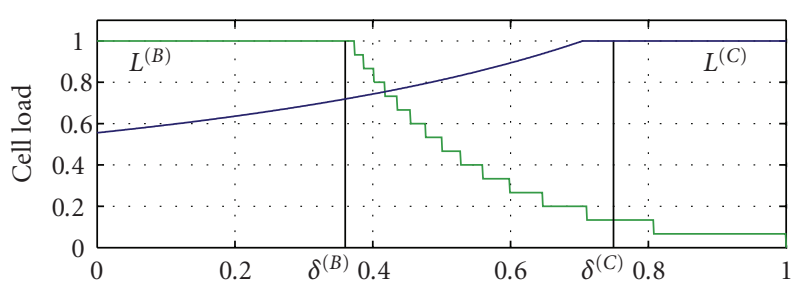

(a)

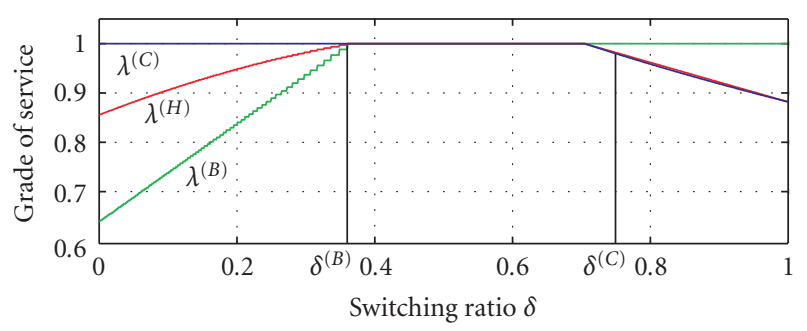

(b)

FIGURE 5: Performance indicators cell load and grade of service in a hybrid network for a single cell, depending on the switching ratio $\delta$.

broadcast network. A significant gain of the GoS exists, if the initial case $\left(\lambda_{\delta=1}^{(C)}\right)$ is compared to compared to the hybrid network with applied unloading $\left(\lambda_{\delta=\delta^{(B)}}^{(H)}\right)$.

In the depicted case, a wide operating range is shown for achieving sufficient GoS. From a network planning point of view, applying an $\delta<\delta^{(B)}$ is not preferable, since the overall $\operatorname{GoS} \lambda^{(H)}$ decreases very fast, due to the high influence of the overloaded broadcast network (see (15)).

\section{Simulation of a Realistic Scenario}

In this section, the behavior of the performance indicators GoS and the cell load is shown by means of a realistic scenario. In the shown example, it is assumed that the broadcast cell covers the entire scenario area. This can be assured by, for example, reusing a DVB-T transmitter for DVB-H, a real transmitter is available at the Berlin scenario.

As mentioned before, the snapshot approach has been used in order to achieve accurate results of the performance indicators. The snapshot method has been implement, considering indoor and outdoor users, and signal shadowing with spatial correlation. We have performed a sufficiently large number of snapshots and tested $t$ confidence interval $[19$, Ch. 4$]$ in order to achieve average values of the transmit power and the GoS, that are within a range of $\pm 1 \%$ around the expected value with a $99 \%$ confidence.

5.1. Simulation Results. For the network scenario, the parameters have been used as described in Section 4.4. The cells of the UMTS network are unloaded, if content is switched from the UMTS network to the broadcast network. The grade of service $\lambda_{i}$ is increased, and the transmit power $p_{i}$ is decreased significantly. 


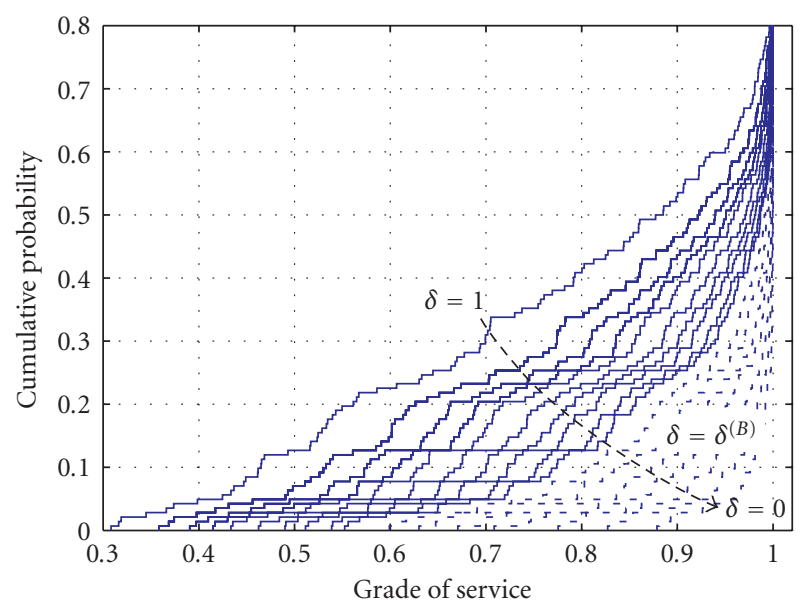

Figure 6: Empirical CDF for grade of service depending on the switching parameter $\delta$. Dotted curves represent the cases with the broadcast network in overload.

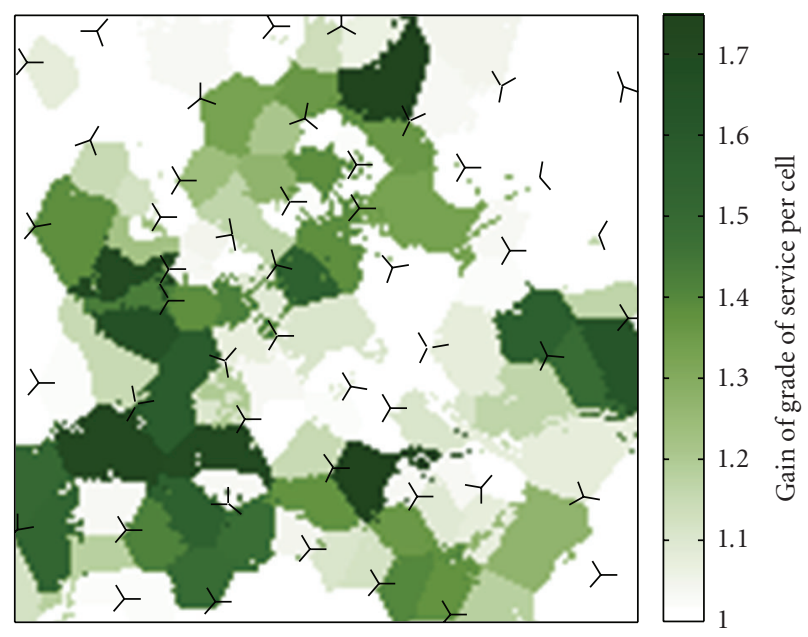

FIGURE 7: Gain of grade of service for unloading the UMTS cells by $\delta=\delta^{(B)}$.

Figure 6 shows the empirical cumulative distribution of the GoS of all cells in the network. Each curve represents a switching value for $\delta$ from zero to one in 0.1 steps and the broadcast bound $\delta^{(B)}=0.36$. It can be seen that the GoS increases for lower values of $\delta$. For the full-loaded network $(\delta=1)$ about $31 \%$ of the cells are above the GoS target $\hat{\lambda}=0.98$. By unloading the cells with $\delta=\delta^{(B)}=0.36$ about $55 \%$ and for $\delta=0$ about $89 \%$ of the cells meet the target threshold. The dotted lines represent the cases with $\delta<\delta^{(B)}$, in which the broadcast network is overloaded.

In order to estimate, which UMTS cells benefit from load switching, the GoS gain is defined by the ratio of the unloaded cells $\left(\lambda_{i, \delta=\delta^{(B)}}^{(C)}\right)$ to the fully loaded case $\left(\lambda_{i, \delta=1}^{(C)}\right)$. Figure 7 shows the gain values, based on the best server cell structure. A high gain occurs for those cells, in which a high traffic demand exists (compare to Figure 1). For the cells with a gain equal to one, unloading has not been necessary due to a low traffic load. A maximum gain of about $75 \%$ increase in

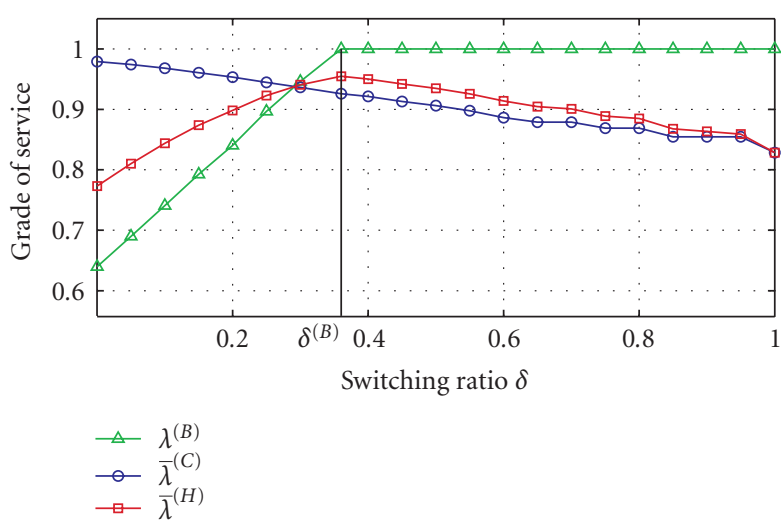

FIGURE 8: Grade of service of the entire network depending on the amount of load switching.

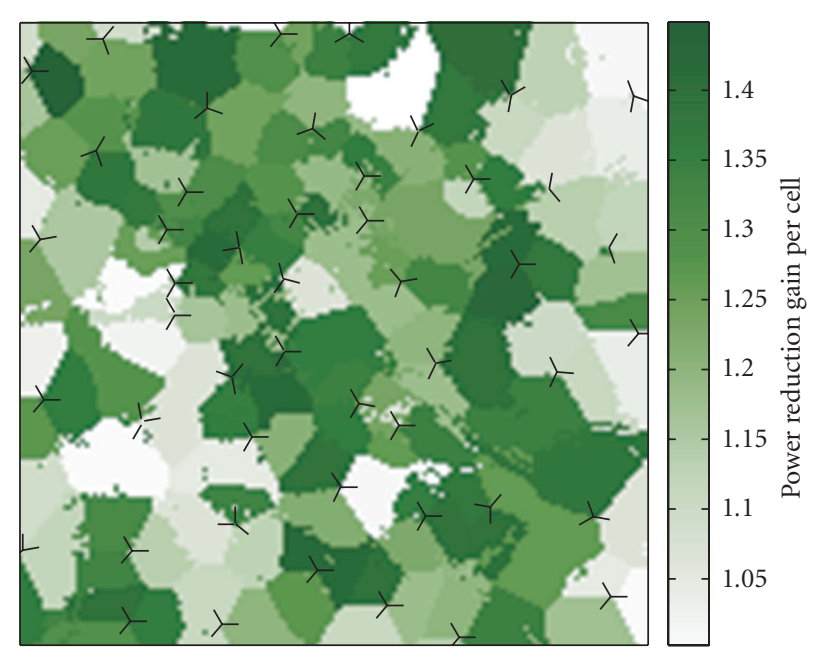

FIGURE 9: Power gain per cell resulting from cell unloading with $\delta=\delta^{(B)}$.

GoS can be seen in some selected cells, which benefit most of using a hybrid mode.

We use (7) in order to calculate the grade of service of the entire network. For network planning the grade of service should be $\hat{\lambda}=98 \%$ or higher. In Figure 8 the performance of the hybrid network is shown. The GoS of the UMTS network $\bar{\lambda}^{(C)}$ increases by unloading but does not meet $\hat{\lambda}$ for an unloading till $\delta^{(B)}\left(\lambda_{\delta=\delta^{(B)}}^{(C)}=92.6 \%\right)$. The UMTS bound $\delta^{(C)}$ is not depicted since for $\delta=0$ a GoS of $\bar{\lambda}^{(C)}=97.9 \%<\hat{\lambda}$ is achieved. Since the $\delta^{(C)}<\delta^{(B)}$ the maximum total GoS occurs for $\delta=\delta^{(B)}$ with $\bar{\lambda}^{(H)}=95.5 \%$.

If cells are unloaded and the GoS target is reached, a further unloading leads to a reduction of the necessary transmit cell power. In Figure 9, the power reduction gain is shown per cell. A maximum reduction gain of 1.45 is achieved. It can be seen that cells with high GoS gain do not show a power gain, since the GoS target has not been reached and thus the power is still at its maximum $p_{\max }$. The complementarity to the GoS can also be seen in this case. 


\section{Conclusion}

We have proposed a system and performance model for hybrid networks, which are composed of the cellular network UMTS and the mobile broadcast network DVB-H. The developed model is based on the existing interference coupling model of the UMTS network, which has been enhanced by the load switching concept. Furthermore, a broadcast system model has been developed. The two network performance indicators cell load and grade of service (GoS) have been defined; whereas the GoS parameter is used as the primary performance criteria for hybrid networks.

Based on the developed models and the GoS definition, the switching bound concept has been been proposed, which define bounds for the load switching for each network separately. They determine thresholds for the necessary unloading of the UMTS network and for the maximum loading of the broadcast network in order to achieve the GoS target. These bounds are used to evaluate the conditions for reasonable load switching and define the necessity and feasibility of hybrid networks. The impact of load switching for the streaming multimedia service has been analyzed by a theoretical and a realistic scenario. We have shown the behavior of the cell load, the grade of service, and the appropriate gain values on a cell basis and for the entire network. The results have shown that a hybrid network can be applied to unload the UMTS network and to serve the multimedia services very efficiently.

\section{References}

[1] U. Reimers, DVB-The Family of International Standards for Digital Video Broadcasting, Springer, Berlin, Germany, 2nd edition, 2005.

[2] ETSI, "Digital Video Broadcasting (DVB); transmission system for handheld terminals (DVB-H)," Tech. Rep. ETSI Std. EN 302304 V1.1.1, ETSI, Sophia Antipolis, France, 2004.

[3] G. Faria, J. A. Henriksson, E. Stare, and P. Talmola, "DVB-H: digital broadcast services to handheld devices," Proceedings of the IEEE, vol. 94, no. 1, pp. 194-209, 2006.

[4] G. May, "Loss-free handover for IP datacast over DVB-H networks," in Proceedings of the International Symposium on Consumer Electronics (ISCE '05), pp. 203-208, June 2005.

[5] G. May and P. Unger, "A new approach for transmitting localized content within digital single frequency broadcast networks," IEEE Transactions on Broadcasting, vol. 4, no. 53, pp. 732-737, 2007.

[6] ETSI, "Digital Video Broadcasting (DVB); IP datacast over DVB-H: architecture," Tech. Rep. TR 102469 V1.1.1, ETSI, Sophia Antipolis, France, May 2006.

[7] M. Kornfeld and G. May, "DVB-H and IP datacast-broadcast to handheld devices," IEEE Transactions on Broadcasting, vol. 53, no. 1, pp. 161-170, 2007.

[8] C. Heuck, Optimierung hybrider (Rundfunk-/Mobilfunk-) Netze durch Steuerung der Lastverteilung, Ph.D. dissertation, Institut für Nachrichtentechnik, TU Braunschweig, Braunschweig, Germany, 2009.

[9] L. Ferreira, L. M. Correia, D. Xavier, I. Vasconcelos, and E. R. Fledderus, "Final report on traffic estimation and services characterisation," Tech. Rep. IST-2000-28088 MOMENTUM, D1.4, IST, 2003, http://momentum.zib.de/ paper/momentum-d14.pdf.
[10] B. Rakoczi, E. R. Fledderus, B. Heideck, P. Lourenço, and T. Kürner, "Reference scenarios," Tech. Rep. IST-2000-28088 MOMENTUM, D5.2, IST, 2003, http://momentum.zib.de/ paper/momentum-d52.pdf.

[11] Momentum Project, IST-2000-28088, Momentum public UMTS planning scenarios, 2003, http://momentum.zib.de/.

[12] T. Kürner, "Propagation models for macro-cells," in Digital Mobile Radio Towards Future Generation Systems (COST 231 Final Report), COST Telecom Secretariat, pp. 134-148, CEC, Brussels, Belgium, 1999, http://www.lx.it.pt/cost231/.

[13] M. Nawrocki, H. Aghvami, and M. Dohler, Eds., Understanding UMTS Radio Network Modelling, Planning and Automated Optimisation: Theory and Practice, John Wiley \& Sons, New York, NY, USA, 2006.

[14] H.-F. Geerdes, UMTS radio network planning: mastering cell coupling for capacity optimization, Ph.D. dissertation, Vieweg and Teubner, Wiesbaden, Germany, 2008.

[15] A. Eisenblätter, H.-F. Geerdes, T. Koch, A. Martin, and R. Wessäly, "UMTS radio network evaluation and optimization beyond snapshots," Mathematical Methods of Operations Research, vol. 63, no. 1, pp. 1-29, 2006.

[16] J. Laiho, A. Wacker, and T. Novosad, Radio Network Planning and Optimisation for UMTS, John Wiley \& Sons, New York, NY, USA, 1st edition, 2002.

[17] C. Heuck and P. Unger, "Load balancing and network planning for DVBH/UMTS hybrid networks," ITS-Europe 2005, Hanover, Germany, June 2005.

[18] J. Aaltonen, Content distribution using wireless broadcast and multicast communication networks, Ph.D. thesis, Tampere University of Technology, Tampere, Finland, 2003.

[19] A. M. Law and D. W. Kelton, Simulation Modelling and Analysis, McGraw-Hill, New York, NY, USA, 2000. 

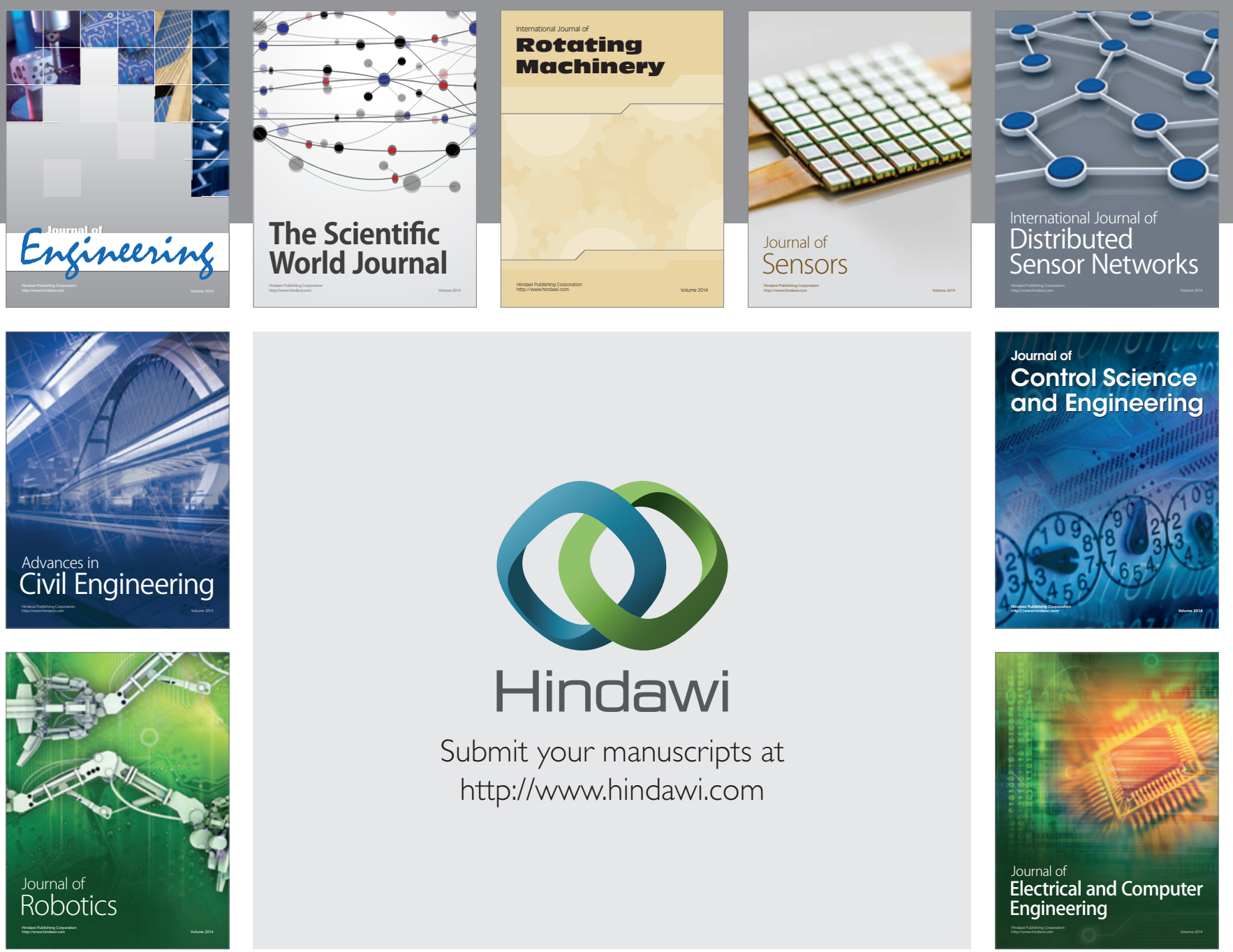

Submit your manuscripts at

http://www.hindawi.com
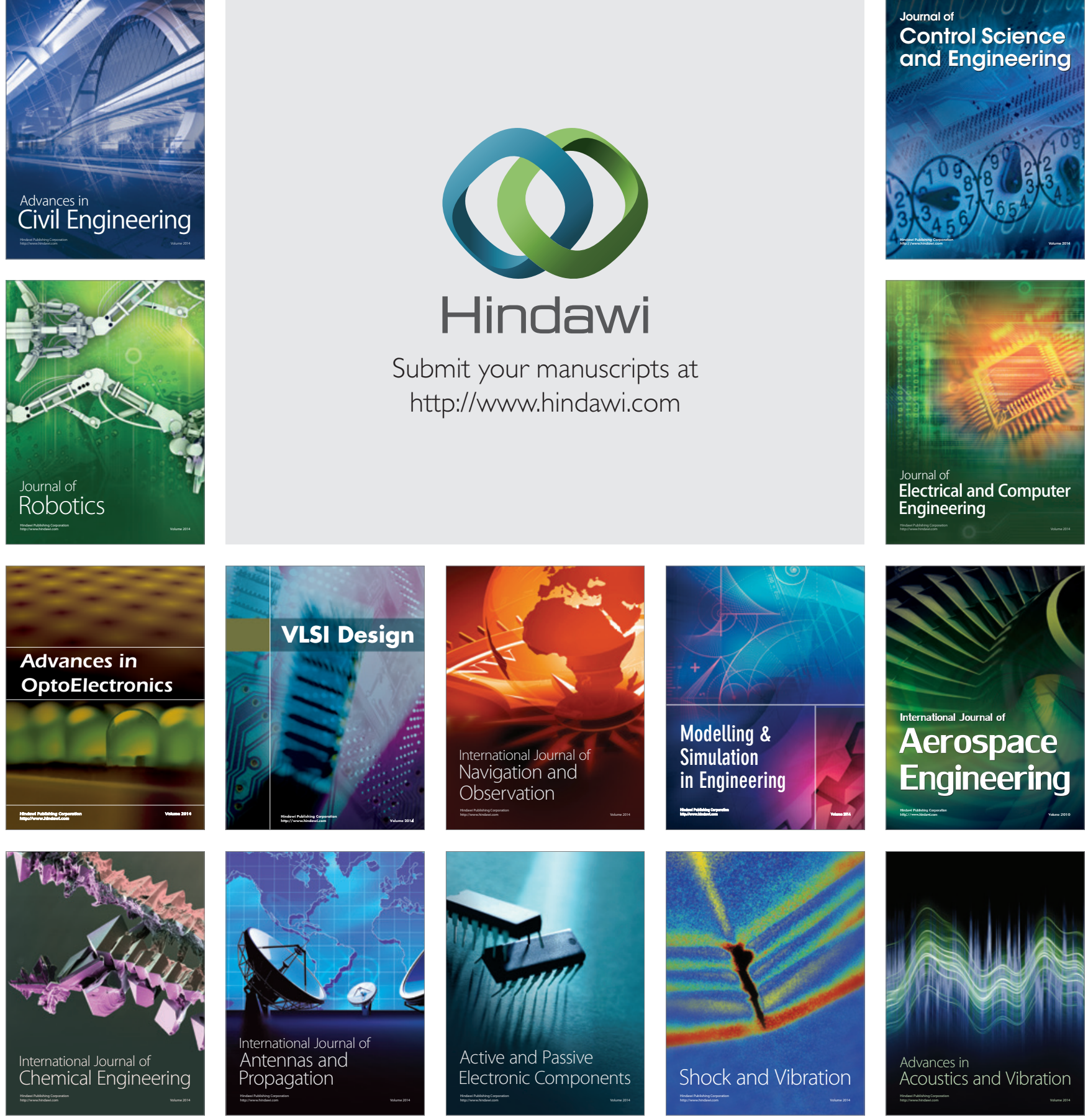